\title{
ON THE MORPHOLOGY OF $\gamma$-EXPANSIONS WITH DELETED DIGITS
}

\author{
MIKE KEANE, MEIR SMORODINSKY AND BORIS SOLOMYAK
}

\begin{abstract}
We investigate the size of the set of reals which can be represented in base $\gamma$ using only the digits $0,1,3$. It is shown that this set has Lebesgue measure zero for $\gamma \leq 1 / 3$ and equals an interval for $\gamma \geq 2 / 5$. Our main goal is to prove that it has Lebesgue measure zero for a certain countable subset of $(1 / 3,2 / 5)$.
\end{abstract}

\section{INTRODUCTION}

In this article we investigate a seemingly simple problem concerning the size of certain compact subsets of the real line. Let us call such a set small if it has Lebesgue measure zero, large if it contains an interval, and intermediate, if it falls between small and large. Palis and Takens (see [PT, p. 151]) have asked whether the difference of two affine Cantor sets is either small or large. Studying this question, the first two authors were led in a natural manner to the following, apparently simpler problem. For each $\gamma$ in the unit interval, let

$$
C_{\gamma}:=\left\{\sum_{n \geq 1} y_{n} \gamma^{n}: \text { for each } n, y_{n} \in\{0,1,3\}\right\} \text {. }
$$

For which $\gamma$ is $C_{\gamma}$ large?

We show first that if $\gamma \leq 1 / 3$, then $C_{\gamma}$ is small, and if $\gamma \geq 2 / 5$, then $C_{\gamma}$ is large. Our principal result is that there is a sequence $\gamma_{k}$ of algebraic integers, all lying between $1 / 3$ and $2 / 5$, such that each $C_{\gamma_{k}}$ is small (in fact, its Hausdorff dimension is less than one). The main tool in the proof is the theory of $\beta$-expansions.

These results were obtained in 1990-92 but for a number of reasons their publication was delayed. In the meantime remarkable progress has been made by Pollicott and Simon [PS] who proved, in particular, that the Hausdorff dimension of $C_{\gamma}$ is equal to one for a.e. $\gamma \in\left(1 / 3, \frac{1}{2}(\sqrt{3}-1)\right)$. In the Appendix we show how a modification of their proof yields the same result for a.e. $\gamma \in(1 / 3,2 / 5)$. Though nothing is implied about the Lebesgue measure, this certainly sheds new light on the problem.

Received by the editors June 2, 1994.

1991 Mathematics Subject Classification. Primary 11K55, 28A78.

Key words and phrases. $\beta$-expansions, Cantor sets, Hausdorff dimension.

Research of the third author was partially supported by NSF grant DMS-9201369. 


\section{ELEMENTARY OBSERVATIONS}

In this section, we show that for $\gamma \geq 2 / 5$ the set $C_{\gamma}$ is large, and for $\gamma \leq 1 / 3$ the set $C_{\gamma}$ is small.

Proposition 1. If $\gamma \geq 2 / 5$, then $C_{\gamma}=\left[0, \frac{3 \gamma}{1-\gamma}\right]$.

Proof. For $x \in\left[0, \frac{3 \gamma}{1-\gamma}\right]$ define

$$
n(x):= \begin{cases}0 & \text { if } 0 \leq x<\gamma \\ 1 & \text { if } \gamma \leq x<3 \gamma \\ 3 & \text { if } 3 \gamma \leq x \leq \frac{3 \gamma}{1-\gamma}\end{cases}
$$

and

$$
T(x):=\frac{1}{\gamma}(x-n(x) \gamma) .
$$

Then

$$
0 \leq T(x) \leq \max \left(2, \frac{3 \gamma}{1-\gamma}\right)=\frac{3 \gamma}{1-\gamma},
$$

since $2 \leq \frac{3 \gamma}{1-\gamma}$ for $\gamma \geq 2 / 5$. Thus under the hypothesis of the proposition, $T$ maps $\left[0, \frac{3 \gamma}{1-\gamma}\right]$ to itself and we are allowed to iterate $T$. Defining

$$
y_{n}:=n\left(T^{n-1} x\right),
$$

one easily shows by induction that

$$
x=y_{1} \gamma+y_{2} \gamma^{2}+\cdots+y_{n} \gamma^{n}+\gamma^{n} T^{n}(x)
$$

for each $n \geq 1$. Therefore

$$
x=\sum_{n=1}^{\infty} y_{n} \gamma^{n} \in C_{\gamma},
$$

as desired.

Proposition 2. If $\gamma \leq 1 / 3$, then $\left|C_{\gamma}\right|=0$.

Proof. Splitting into the three possible values for $y_{1}$, we see immediately that

$$
C_{\gamma}=\gamma C_{\gamma} \cup\left(\gamma+\gamma C_{\gamma}\right) \cup\left(3 \gamma+\gamma C_{\gamma}\right) .
$$

Subadditivity and scaling properties of Lebesgue measure then produces

$$
\left|C_{\gamma}\right| \leq 3 \gamma\left|C_{\gamma}\right|
$$

thus if $\gamma<1 / 3$, then $\left|C_{\gamma}\right|$ must be zero. A slight modification now yields the required result for $\gamma=1 / 3$, as follows. In the representation of $C_{\gamma}$ as a union of three sets above, the set $3 \gamma+\gamma C_{\gamma}$ is disjoint from the other two sets of the union, since

$$
\gamma+\sum_{n=2}^{\infty} 3 \gamma^{n}=\gamma+\frac{3 \gamma^{2}}{1-\gamma}<3 \gamma
$$

for any $\gamma<2 / 5$, hence also for $\gamma=1 / 3$. Setting

$$
I:=\gamma C_{\gamma} \cap\left(\gamma+\gamma C_{\gamma}\right)
$$


now leads to

$$
\left|C_{\gamma}\right|=3 \gamma\left|C_{\gamma}\right|-|I|,
$$

which for $\gamma=1 / 3$ implies $|I|=0$. But the set

$$
\gamma+\gamma^{2} C_{\gamma}=3 \gamma^{2}+\gamma^{2} C_{\gamma}
$$

is contained in $I$ if $\gamma=1 / 3$, since then $3 \gamma^{2}=\gamma$. Thus

$$
\left|\gamma+\gamma^{2} C_{\gamma}\right|=\gamma^{2}\left|C_{\gamma}\right|=0
$$

as required, if $\gamma=1 / 3$.

2. Parameter values Between $1 / 3$ and $2 / 5$

For $k \geq 1$, we denote by $\gamma_{k}$ the unique solution of

$$
1=2 \gamma+2 \gamma^{2}+\cdots+2 \gamma^{k}
$$

lying in the unit interval. If $k=1$, then $\gamma_{1}=1 / 2$ and by Proposition $1, C_{\gamma_{1}}$ is large. For $k \geq 2, \gamma_{k} \in(1 / 3,2 / 5)$ and one sees easily that

$$
1 / 3<\cdots<\gamma_{3}<\gamma_{2}<2 / 5
$$

with

$$
\lim _{k \rightarrow \infty} \gamma_{k}=1 / 3 .
$$

Proposition 3. For $k \geq 3,\left|C_{\gamma_{k}}\right|=0$.

Proof. Fix $k \geq 3$ and set $\gamma:=\gamma_{k}$. We have seen in the proof of Proposition 2 that

$$
\left|C_{\gamma}\right|=3 \gamma\left|C_{\gamma}\right|-|I|,
$$

with

$$
I=\gamma C_{\gamma} \cap\left(\gamma+\gamma C_{\gamma}\right) .
$$

In this case, however, $3 \gamma>1$, so that we shall need to find several scaled copies of $C_{\gamma}$ in $I$ in order to conclude that $\left|C_{\gamma}\right|=0$. We adopt the simple notation

$$
y_{1} \ldots y_{n}:=y_{1} \gamma+\cdots+y_{n} \gamma^{n} \text {. }
$$

Then by definition

$$
1=\cdot \underbrace{22 \ldots 2}_{k \text { times }} \text {, }
$$

so that

$$
.0 \underbrace{33 \ldots 3}_{k \text { times }}=. \underbrace{111 \ldots 1}_{k+1 \text { times }} .
$$

This shows that

$$
\cdot \underbrace{11 \ldots 1}_{k+1 \text { times }}+\gamma^{k+1} C_{\gamma} \subseteq I .
$$

Furthermore,

$$
.3=\cdot \underbrace{22 \ldots 2}_{k+1 \text { times }}=1 \cdot \underbrace{00 \ldots 2}_{k \text { times }} \text {, }
$$


so that

$$
\begin{aligned}
& .030 \ldots 01=.100 \ldots 03, \\
& .030 \ldots 11=.100 \ldots 13, \\
& .030 \ldots 31=.100 \ldots 33
\end{aligned}
$$

(each number containing $k+2$ 'digits'), and hence the sets

$$
\begin{aligned}
& .100 \ldots 03+\gamma^{k+2} C_{\gamma}, \\
& .100 \ldots 13+\gamma^{k+2} C_{\gamma}, \\
& .100 \ldots 33+\gamma^{k+2} C_{\gamma}
\end{aligned}
$$

are subsets of $I$. We claim that these four subsets of $I$ are mutually disjoint if $k \geq 3$, leaving the simple calculations necessary to show this to the reader. Therefore

$$
\left|C_{\gamma}\right|=3 \gamma\left|C_{\gamma}\right|-|I| \leq 3 \gamma\left|C_{\gamma}\right|-\gamma^{k+1}\left|C_{\gamma}\right|-3 \gamma^{k+2}\left|C_{\gamma}\right| .
$$

If now $\left|C_{\gamma}\right|>C$, then it follows that

$$
1 \leq 3 \gamma-\gamma^{k+1}-3 \gamma^{k+2} \text {. }
$$

But $3 \gamma-1=2 \gamma^{k+1}$, so we obtain finally $\gamma^{k+1} \geq 3 \gamma^{k+2}$ and $\gamma \leq 1 / 3$, a contradiction. Hence $\left|C_{\gamma}\right|=0$.

Proposition 4. $\left|C_{\gamma_{2}}\right|=0$.

Our proof of this proposition is rather long, and in one place mysterious. As it is not our intention to lose the reader during the calculations which follow, we try to explain what is going to happen first. We must consider all numbers

$$
y_{1} y_{2} y_{3} \cdots=\sum_{n=1}^{\infty} y_{n} \gamma^{n}
$$

as above, where $y_{n} \in\{0,1,3\}$ for each $n$ and $\gamma=\gamma_{2}$ satisfies

$$
1=2 \gamma+2 \gamma^{2}
$$

or, equivalently,

$$
1=.22 \text {. }
$$

All notations with a point are interpreted 'base gamma'. For any real number $x$, there is a canonical gamma expansion. We shall only be interested in values of $x$ between 0 and $1 / \gamma$; in this case we can write

$$
x=x_{0} \cdot x_{1} x_{2} \ldots
$$

with $x_{n} \in\{0,1,2\}$ and $x_{n} x_{n+1} \neq 22$ for each $n \geq 0$. The advantage of this expansion is that it is essentially unique, in the same way that the ordinary decimal expansion is unique. Thus, given any sequence $y_{1}, y_{2}, \cdots \in\{0,1,3\}$, we can find $x_{0}, x_{1}, \ldots$ as above such that

$$
y_{1} y_{2} \cdots=x_{0} \cdot x_{1} x_{2} \ldots
$$

In principle, given any $x$ it is possible to calculate one by one symbols $y_{n} \in$ $\{0,1,3\}$ in order to express $x$ as an expansion in these digits; sometimes this calculation branches (i.e., we may be able to use either $y_{n}=0$ or $y_{n}=1$ at stage $n$ ), and sometimes it dies, showing that $x$ does not belong to $C_{\gamma}$. Just as 
in the case of the decimal expansion, the gamma expansion is ergodic. That is, if we specify some admissible sequence $b=b_{1} \ldots b_{l}$ of zeroes, ones, and twos (admissible meaning that $b_{i} b_{i+1} \neq 22$ for each $i$ ), then the set of $x$ whose canonical expansion does not contain $b$ has Lebesgue measure zero. Thus in order to prove the proposition, it is sufficient to find a fixed admissible block $b$ such that for any given $y$-sequence, the block $b$ does not occur anywhere in the canonical expansion of this sequence. The mysterious part of the proof is that we have found such a block; it is

$$
b=20000200020002 \text {. }
$$

In order to show that for a fixed $n$,

$$
x_{n} x_{n+1} \ldots x_{n+13} \neq b
$$

in the canonical expansion of any $y$-sequence, we proceed by splitting the $y$ sequence into two parts:

$$
y_{1} y_{2} \ldots=. y_{1} \ldots y_{n}+.0 \ldots 0 y_{n+1} y_{n+2} \ldots
$$

First we calculate the effect which the initial part will have on the canonical expansion at the places $n, n+1, \ldots$. This reduces the problem to finitely many cases, which are then examined separately to show that the given block $b$ is impossible in each case, using the branching procedure alluded to above.

We now begin by stating the properties of the canonical expansion, which can be found in $[\mathrm{P}, \mathrm{R}, \mathrm{S}]$. They are all relatively easy to derive in the case $1=2 \gamma+2 \gamma^{2}$ under consideration.

Property 1. For each $x \in[0,1 / \gamma]$ there exists an admissible sequence $x_{0}, x_{1}$, $\cdots \in\{0,1,2\}$ (admissible meaning that $x_{n} x_{n+1} \neq 22$ for each $n \geq 0$ ) such that

$$
x=x_{0} \cdot x_{1} x_{2} \ldots
$$

Property 2. Except for countably many $x \in[0,1 / \gamma]$, the admissible sequence in Property 1 is unique. The exceptions are exactly those $x$ which have finite expansions, and they have exactly two representations (except for $x=0$ ) :

$$
x=x_{0} \cdot x_{1} \ldots x_{k}=x_{0} \cdot x_{1} \ldots\left(x_{k}-1\right) 212121 \ldots
$$

Property 3. If $b$ is any admissible block, the set of $x \in[0,1 / \gamma]$ whose admissible sequences do not contain $b$ at any place in their expansions has Lebesgue measure zero.

In the sequel, we call $x_{0} \cdot x_{1} x_{2} \ldots$ the canonical expansion of $x$, using the finite version for the exceptions stated in Property 2.

Now we fix a positive integer $n$, and begin to calculate the influence of the first $n \quad y$-values.

Lemma 1. If $y_{1}, \ldots, y_{n} \in\{0,1,3\}$, then the canonical expansion of . $y_{1} \ldots y_{n}$ is of the form

$$
x_{0} \cdot x_{1} \ldots x_{n} x_{n+1} x_{n+2}
$$

(i.e., $x_{k}=0$ for $k \geq n+3$ ). Moreover, the triple $x_{n} x_{n+1} x_{n+2}$ belongs to the following list:

$$
L=\{000,002,020,100,120,200,202\} .
$$


Proof. For $n=1$ there are three possibilities:

$$
.0=.000, .1=.100, \quad .3=1.002,
$$

the last one coming from the equality

$$
.3=.222=1.002 \text {. }
$$

(Although one does get better at gamma arithmetic with practice, it remains surprisingly difficult to accomplish elementary manipulations in some cases!) Now proceed by induction, supposing that the lemma is valid for $n-1$ and considering the three cases for $y_{n}$. The following table gives the new values $x_{n} x_{n+1} x_{n+2}$ as functions of the old values $x_{n-1} x_{n} x_{n+1}$ :

$\begin{array}{llll} & y_{n}=0 & y_{n}=1 & y_{n}=3 \\ \text { old } & \text { new } & \text { new } & \text { new } \\ 000 & 000 & 100 & 002 \\ 002 & 020 & 120 & 100 \\ 020 & 200 & 002 & 202 \\ 100 & 000 & 100 & 002 \\ 120 & 200 & 002 & 002 \text { or } 202 \\ 200 & 000 & 100 & 100 \\ 202 & 020 & 120 & 120\end{array}$

Again, some dexterity in calculation is necessary. The ambiguous case in which $y_{n}=3$ and $x_{n-1} x_{n} x_{n+1}=120$ is instructive.

Lemma 2. Let $y_{1}, y_{2}, \cdots \in\{0,1,3\}$ and let

$$
x:=y_{1} y_{2} \cdots=x_{0} \cdot x_{1} x_{2} \ldots
$$

be the associated canonical expansion. Let $n \geq 1$ be fixed, and denote by

$$
\hat{x}:=. y_{1} \ldots y_{n}=\hat{x}_{0} \cdot \hat{x}_{1} \ldots \hat{x}_{n+2}
$$

the canonical expansion of the first part of $y$. Suppose furthermore that $x_{n}=2$. Then $\hat{x}_{i}=x_{i}$ for each $0 \leq i \leq n-1$.

Proof. On the one hand,

$$
x-\hat{x}=.0 \ldots 0 y_{n+1} y_{n+2} \cdots \leq .0 \ldots 033 \cdots=\gamma^{n}+2 \gamma^{n+1} .
$$

On the other hand, if $i$ is the least integer between 0 and $n-1$ for which $\hat{x}_{i} \neq x_{i}$, then $x_{i}>\hat{x}_{i}$ and

$$
x=x_{0} \cdot x_{1} x_{2} \ldots \geq x_{0} \cdot x_{1} \ldots x_{i} 0 \ldots 0 x_{n} 0 \ldots
$$

(where $x_{n}=2$ by hypothesis) and

$$
\hat{x}=\hat{x}_{0} \cdot \hat{x}_{1} \hat{x}_{2} \ldots \leq x_{0} \cdot x_{1} \ldots x_{i} .
$$

Therefore $x-\hat{x} \geq 2 \gamma^{n}$, leading to $2 \gamma^{n} \leq \gamma^{n}+2 \gamma^{n+1}$ and $1 \leq 2 \gamma$, a contradiction.

Corollary 1. Under the hypotheses and notation of Lemma 2,

$$
. y_{n+1} y_{n+2} \cdots+a \cdot b c=x_{n} \cdot x_{n+1} x_{n+2} \cdots
$$

for some $a b c \in L$ (the list of Lemma 1 ).

Proof. Use Lemmas 1 and 2, and multiply by $\gamma^{-n}$.

This concludes the calculation of the effect the initial part has on the canonical expansion at place $n$, and we can now begin with the examination of cases. 
Lemma 3. If $y_{1}, y_{2} \cdots \in\{0,1,3\}$ with

$$
x=y_{1} y_{2} \cdots=x_{0} \cdot x_{1} x_{2} \ldots
$$

being its canonical expansion, then for any $n \geq 0$,

$$
x_{n} x_{n+1} \ldots x_{n+13} \neq 20000200020002 \text {. }
$$

Proof. Suppose that the remarkable block did appear at place $n$. Then by using Corollary 1 and changing the indices, there must exist $y_{1}, y_{2}, \ldots$ and $a b c \in L$ such that

$$
. y_{1} y_{2} \cdots+a . b c=2.0000200020002 \ldots \text {. }
$$

We now show this to be impossible separately for each element of $L$. In order to determine which values for the $y_{i}$ are possible, note first that corresponding to the three possible values 0,1 , and 3 of $y_{1}$, the point.$y_{1} y_{2} \ldots$ must lie in the respective intervals

$$
I_{0}=[0, .12], I_{1}=[.1,1.0], I_{3}=[1.002,1.2] .
$$

This shows immediately that the values 000 and 002 for $a b c$ are impossible, since then the left-hand side would be too small, while also $a b c=202$ is seen to be too large. There remain four possible values for $a b c$.

Case 1. Suppose $a b c=100$. This leads to

$$
y_{1} y_{2} \ldots=1.0000200020002 \ldots
$$

by subtraction, but the right-hand side falls between the intervals $I_{1}$ and $I_{3}$. Hence this case is impossible.

Case 2. Suppose $a b c=200$. Then we must have

$$
y_{1} y_{2} \cdots=0.0000200020002 \ldots
$$

Examining the possible intervals above and shifting (i.e., multiplying by $1 / \gamma$ ) successively, we conclude that $y_{1}=y_{2}=y_{3}=y_{4}=0, y_{5}=1$, and

$$
. y_{6} y_{7} \cdots=1.00020002 \ldots \text {, }
$$

which is impossible, again falling between $I_{1}$ and $I_{3}$.

Case 3. Suppose $a b c=120$. Then

$$
y_{1} y_{2} \cdots=2.0000200020002 \cdots-1.2=.0200200020002 \ldots,
$$

and successively we obtain $y_{1}=0, y_{2}=1, y_{3}=3, y_{4}=y_{5}=y_{6}=y_{7}=y_{8}=$ $0, y_{9}=1$, with

$$
y_{10} y_{11} \cdots=1.0002 \ldots \text {, }
$$

again between $I_{1}$ and $I_{3}$, hence impossible.

In the remaining case, some branching will occur, as both 0 and 1 will be possible at some point.

Case 4. Suppose $a b c=020$. Then

$$
. y_{1} y_{2} \cdots=2.0000200020002 \cdots-0.2=1.0200200020002 \ldots \text {. }
$$

This first implies that $y_{1}=3$ and

$$
. y_{2} y_{3} \cdots=.110000020002 \ldots
$$

Now either $y_{2}=0$ (first branch) or $y_{2}=1$ (second branch) are possible. 
First branch:

$$
y_{3} y_{4} \cdots=1.10000020002 \ldots,
$$

so that $y_{3}=3, y_{4}=1$, and $. y_{5} y_{6} \cdots=1.000020002 \ldots$, which is impossible.

Second branch:

$$
y_{3} y_{4} \cdots=0.1000020002 \ldots
$$

leads to branching again, both $y_{3}=0$ and $y_{3}=1$ being possible. But $y_{3}=0$ leads immediately to

$$
y_{4} y_{5} \cdots=1.000020002 \ldots \text {, }
$$

which is impossible, and $y_{3}$ produces $y_{4}=y_{5}=y_{6}=y_{7}=0, y_{8}=1$, and

$$
y_{9} y_{10} \cdots=1.0002 \text {, }
$$

which again falls into the forbidden gap.

Proof of Proposition 4. This now follows immediately from Lemma 3 and the ergodic Property 3.

\section{CONCLUDING REMARKS, GENERALIZATIONS, OPEN QUESTIONS}

In $\S 2$ it is demonstrated that for a sequence $\gamma_{k}, k \geq 2$, converging to $1 / 3$, the set $C_{\gamma_{k}}$ is small. Two different methods were used. For $k \geq 3$ we used what can be called the method of common blocks which led to estimates of $\left|\gamma C_{\gamma} \cap\left(\gamma+\gamma C_{\gamma}\right)\right|$ from below. For $k=2$ we used the method of forbidden blocks which involved finding a block admissible for a general $\gamma$-representation, but forbidden in representations of the elements of $C_{\gamma}$.

Remarks. (1) Let $\hat{\gamma}=(3-\sqrt{5}) / 2=.381966 \ldots$. The canonical $\hat{\gamma}$-expansion of 1 is $.2111 \ldots$, and in this base we have $.3=1.01$. We have proved that $C_{\hat{\gamma}}$ is small by showing that the block 20020020112002 is forbidden. This is the largest $\gamma \in(1 / 3,2 / 5)$ for which we know the answer. The argument is similar to the one in Proposition 4, but quite a bit longer.

(2) The method of common blocks can be used to show that $C_{\gamma}$ is small in many other cases, when $\gamma$ is sufficiently close to $1 / 3$. We have the following:

Proposition 5. Let $\gamma \in(1 / 3,2 / 5)$ be such that the canonical $\gamma$-expansion of 1 is:

$$
1 .=d_{1} d_{2} d_{3} \ldots, \quad \text { where } d_{1}=\cdots=d_{k}=2
$$

(it may be finite or infinite). Suppose that the number of "digit changes" $d_{j}-$ $d_{j+1} \neq 0$ is finite and equal to $m$ (in particular, this implies that $d_{j}$ are eventually fixed). If $m \leq k-2$, then the set $C_{\gamma}$ is small.

This proposition implies Proposition 3 (where $m=1$ ) and gives many other examples: for instance, $\gamma$ corresponding to

$$
1 .=.2222 \underbrace{11 \ldots 1}_{k \text { times }}, \quad k=1,2, \ldots, \infty .
$$

The set of $\gamma$ covered by Proposition 5 has a countable set of limit points.

Sketch of the proof. It follows from the canonical representation of 1 that

$$
\text { 3. }=2 . d_{1} d_{2} \ldots=10 .\left(d_{1}-d_{2}\right)\left(d_{2}-d_{3}\right) \ldots
$$


(using that $d_{1}=2$ ). Consider the set

$$
\mathscr{L}=\left\{x=3+\sum_{j=1}^{\infty} b_{j} \gamma^{j}=\gamma^{-1}+\sum_{j=1}^{\infty}\left(d_{j}-d_{j+1}+b_{j}\right) \gamma^{j}\right\},
$$

where

$$
b_{j}:= \begin{cases}0 & \text { if } d_{j}-d_{j+1}=1, \\ 1 & \text { if } d_{j}-d_{j+1}=-1 \text { or } 2, \\ 3 & \text { if } d_{j}-d_{j+1}=-2, \\ 0,1 \text { or } 3 & \text { if } d_{j}-d_{j+1}=0 .\end{cases}
$$

The choice of $b_{j}$ implies that $\gamma^{2} \mathscr{L} \subset \gamma C_{\gamma} \cap\left(\gamma+\gamma C_{\gamma}\right)=: I$.

Let us estimate $|\mathscr{L}|$ from below. Observe that the sum $\sum_{j=1}^{\infty} b_{j} \gamma^{j}$ in the definition of $\mathscr{L}$ is an arbitrary element of $C_{\gamma}$, with $m$ fixed digits (for $j$ such that $d_{j} \neq d_{j+1}$ ). Clearly, making a different choice of these $m$ digits results in a set which is a translation of $\mathscr{L}$. Thus, $C_{\gamma}$ is a union of $3^{m}$ translated copies of $\mathscr{L}$, and therefore

$$
|\mathscr{L}| \geq 3^{-m}\left|C_{\gamma}\right|
$$

As in the proof of Propositions 2 and 3, we can write

$$
\left|C_{\gamma}\right|=3 \gamma\left|C_{\gamma}\right|-|I| \leq 3 \gamma\left|C_{\gamma}\right|-\gamma^{2}|\mathscr{L}| \leq\left(3 \gamma-3^{-m} \gamma^{2}\right)\left|C_{\gamma}\right| .
$$

Thus,

$$
\gamma\left(3-\gamma^{-1}-3^{-m} \gamma\right)\left|C_{\gamma}\right| \geq 0 .
$$

However, $\gamma \leq \gamma_{k}$ (where $\gamma_{k}$ satisfies $1=2 \gamma_{k}+\cdots+2 \gamma_{k}^{k}$ ), so

$$
3-\gamma^{-1} \leq 3-\gamma_{k}^{-1}=2 \gamma_{k}^{k}
$$

Working in base $\gamma_{k}$ we obtain

$$
1 .=. \underbrace{22 \ldots 2}_{k \text { times }}>.0 \underbrace{66 \ldots 6}_{k-1 \text { times }}>\cdots>\cdot \underbrace{00 \ldots 0}_{k-1 \text { times }}\left(2 \cdot 3^{k-1}\right)=2 \cdot 3^{k-1} \gamma_{k}^{k} .
$$

Using this and keeping in mind that $\gamma>1 / 3$, we get for $m \leq k-2$ :

$$
3-\gamma^{-1}-3^{-m} \gamma<3^{-k+1}-3^{-m-1} \leq 0 .
$$

We conclude that $\left|C_{\gamma}\right|=0$, as desired.

Hausdorff dimension. Let $h(\gamma)$ be the Hausdorff dimension of the set $C_{\gamma}$. What can be said about the behavior of $h(\gamma)$ as a function of $\gamma$ ? Obviously $h(\gamma)=1$ for $\gamma \geq 2 / 5$, and it is easy to see that $h(\gamma)=\log 3 / \log \frac{1}{\gamma}$ for $\gamma \leq 1 / 4$.

In a recent paper by Pollicott and Simon [PS] it is proved that

$$
h(\gamma)=\log 3 / \log \frac{1}{\gamma} \quad \text { for a.e. } \gamma \in(1 / 4,1 / 3),
$$

but

$$
h(\gamma)<\log 3 / \log \frac{1}{\gamma} \quad \text { for } \quad \gamma \in \mathscr{E},
$$

where $\mathscr{E}$ is dense in $(1 / 4,1 / 3)$. Moreover, Pollicott and Simon show that

$$
h(\gamma)=1 \text { for a.e. } \gamma \in\left(1 / 3, \gamma_{2}\right)=\left(1 / 3, \frac{1}{2}(\sqrt{3}-1)\right) \text {. }
$$

In fact, it is possible to modify the argument of [PS] to demonstrate that $h(\gamma)=$ 1 for a.e. $\gamma \in(1 / 3,2 / 5)$ (see Appendix). 
The proofs of Propositions 3, 4 and 5 can be easily adapted to show that $h(\gamma)<1$ for the values of $\gamma$ involved. This is done by considering the $s$ dimensional Hausdorff measure for $s<1$ instead of the Lebesgue measure.

We conclude with some problems which we have not been able to settle.

Problem 1. Is it true that $\left|C_{\gamma}\right|>0$ for a.e. $\gamma \in(1 / 3,2 / 5) ?^{1}$

Problem 2. Is there $\gamma$ such that $C_{\gamma}$ is intermediate?

Problem 3. Investigate the "exceptional set" of $\gamma \in(1 / 3,2 / 5)$ such that $h(\gamma)<$ 1. Is it dense?

\section{APPENDIX}

Proposition 6. For a.e. $\gamma \in(1 / 3,2 / 5)$ with respect to the Lebesgue measure, we have $h(\gamma)=1$.

This result for $\gamma \in\left(1 / 3, \frac{1}{2}(\sqrt{3}-1)\right)=\left(1 / 3, \gamma_{2}\right)$ was obtained by Pollicott and Simon [PS, 4(1)]. We are using their scheme with some modifications to get this for $\gamma \in\left(\gamma_{2}, 2 / 5\right)$. Therefore we will refer to [PS] extensively, indicating only the changes that have to be made. Here we only deal with the $(0,1,3)$-case, however, similar ideas can be applied to a more general situation of expansions with deleted digits considered in [PS].

Proof. Let $\Sigma=\{0,1,3\}^{\mathbb{N}}$ be the one-sided full shift on the symbols $0,1,3$, and set

$$
\Pi^{\gamma}\left(s_{1} s_{2} \ldots\right):=\sum_{n=1}^{\infty} s_{n} \gamma^{n}, \quad \text { for } s=\left(s_{1} s_{2} \ldots\right) \in \Sigma .
$$

Clearly, $\Pi^{\gamma}$ maps $\Sigma$ onto $C_{\gamma}$. Next consider the one-sided shift of finite type

$$
\Sigma^{\prime}=\left\{s \in \Sigma: s_{i} s_{i+1} \neq 33\right\},
$$

and let $C_{\gamma}^{\prime}=\Pi^{\gamma}\left(\Sigma^{\prime}\right) \subset C_{\gamma}$. We are going to prove that the Hausdorff dimension $h^{\prime}(\gamma)$ of $C_{\gamma}^{\prime}$ is equal to one, for a.e. $\gamma \in\left(\gamma_{2}, 2 / 5\right)$.

First we remark that the topological entropy of $\Sigma^{\prime}$ is exactly $\log \left(1 / \gamma_{2}\right)$. This follows by a straightforward calculation: the matrix associated with $\Sigma^{\prime}$ has the largest eigenvalue $1+\sqrt{3}=1 / \gamma_{2}$. Another way to see this is to note that $\Sigma^{\prime}$ is isomorphic to the $\beta$-shift for $\beta=1 / \gamma_{2}$, and it it is well-known (see [W]) that the $\beta$-shift has topological entropy $\log \beta$. (Recall that $1 .=.22$ in base $\gamma_{2}$, and so this $\beta$-shift consists of $(0,1,2)$-sequences with the only forbidden block 22.)

By the definition of topological entropy, the number of $n$-blocks in $\Sigma^{\prime}$ has the order of $\gamma_{2}^{-n}$. This makes it plausible that $\gamma_{2}$ is the critical parameter for $h^{\prime}(\gamma)$. In fact, one can prove that $h^{\prime}(\gamma)=\log \left(\gamma_{2}\right) / \log (\gamma)$ for a.e. $\gamma<\gamma_{2}$.

In order to realize the scheme of [PS, Theorem 1 and 4(1)], we need to find a measure $\nu_{\gamma}$ on $C_{\gamma}^{\prime}$ such that for any $\epsilon>0$,

$$
\int_{\gamma_{2}+\varepsilon}^{2 / 5} \int_{C_{\gamma}^{\prime}} \int_{C_{y}^{\prime}} \frac{d \nu_{\gamma}(x) d \nu_{\gamma}(y)}{|x-y|^{1-\epsilon}} d \gamma<\infty .
$$

${ }^{1}$ Added in proof. It was recently shown that the answer is "yes", see B. Solomyak, On the random series $\sum \pm \lambda^{n}$ (an Erdös problem), to appear in Ann. of Math. 
Then Fubini's theorem and the potential-theoretic characterization of the Hausdorff dimension imply the desired result.

Let $\mu$ be the measure of maximal entropy on $\Sigma^{\prime}$ (see [W]). All we need is that the measure of a cylinder set $\mu\left[s_{1} s_{2} \ldots s_{k}\right]$ has the order of $\gamma_{2}^{k}$. Then set $\nu_{\gamma}$ to be the projection of $\mu: \nu_{y}:=\left(\Pi^{\gamma}\right)^{*} \mu$. The key ingredient of the proof is the following lemma which is the analog of [PS, Lemma 1]. Let

$$
f(\gamma, s, t):=\Pi^{\gamma}(s)-\Pi^{\gamma}(t)=\sum_{n=1}^{\infty}\left(s_{i}-t_{i}\right) \gamma^{i}
$$

Lemma 4. There exists $\delta>0$ such that for all $s, t \in \Sigma^{\prime}$ with $s_{1} \neq t_{1}$, and all $\gamma \in\left(\gamma_{2}, 2 / 5\right)$,

$$
\text { if }|f(\gamma, s, t)|<\delta, \text { then }\left|\frac{d}{d \gamma} f(\gamma, s, t)\right|>\delta .
$$

Proof of Lemma 4. Following [PS, Lemma 1], we can write

$$
\begin{aligned}
& \left|\frac{2 f(\gamma, s, t)}{\gamma}-f^{\prime}(\gamma, s, t)\right| \\
& \quad \geq\left|s_{1}-t_{1}\right|-\left|\sum_{n=3}^{\infty}(n-2)\left(s_{n}-t_{n}\right) \gamma^{n-1}\right| \geq 1-|g(\gamma)|,
\end{aligned}
$$

where $g(\gamma):=\sum_{n=3}^{\infty}(n-2)\left(s_{n}-t_{n}\right) \gamma^{n-1}$. Here we used that $s_{1} \neq t_{1}$. Without loss of generality, one can assume that $g(\gamma) \geq 0$. Then the maximum of $g(\gamma)$ is clearly attained when $s_{3} s_{4} s_{5} \ldots=3131 \ldots$ and $t_{3} t_{4} t_{5} \ldots=000 \ldots$ A direct computation yields

$$
\begin{aligned}
|g(\gamma)| & \leq \sum_{n=3}^{\infty}(n-2) s_{n} \gamma^{n-1} \leq \sum_{k=1}^{\infty}(2 k-1) 3 \gamma^{2 k}+\sum_{k=1}^{\infty} 2 k \gamma^{2 k+1} \\
& =\frac{\gamma^{2}\left(3+2 \gamma+3 \gamma^{2}\right)}{\left(1-\gamma^{2}\right)^{2}} .
\end{aligned}
$$

The last expression is increasing in $\gamma$ and equals $428 / 441<1$ for $\gamma=2 / 5$. The lemma follows.

The rest of the proof proceeds exactly as in [PS, Theorem 1 and 4(1)].

\section{REFERENCES}

[P] W. Parry, On the B-expansion of real numbers., Acta Math. Acad. Sci. Hungar. 11 (1960), 401-416.

[PS] M. Pollicott and K. Simon, The Hausdorff dimension of $\lambda$-expansions with deleted digits, Trans. Amer. Math. Soc. 347 (1995), 967-983.

[PT] J. Palis and F. Takens, Hyperbolicity and sensitive chaotic dynamics at homoclinic bifurcations, Cambridge Univ. Press, Cambridge, 1993.

[R] A. Renyi, Representations for real numbers and their ergodic properties., Acta Math. Acad. Sci. Hungar. 8 (1957), 472-493. 
[S] M. Smorodinsky, B-automorphisms are Bernoulli shifts, Acta Math. Acad. Sci. Hungar. 24 (1973), 273-278.

[W] P. Walters, An introduction to ergodic theory, Springer-Verlag, New York, 1982.

CWI, Post Office Box 4079, 1009 AB Amsterdam and Delft University of Technology, Mekelweg 4, 2628 CD Delft, The Netherlands

E-mail address: keane@cri.nl

School of Mathematical Sciences, Tel Aviv University, Tel Aviv, Israel

E-mail address: meir@math.tau.ac.il

Department of Mathematics, GN-50, University of Washington, Seattle, Wa 98195

E-mail address: solomyak@math. washington.edu 